\title{
Recurrence and azimuthal-symmetry breaking of a cylindrical Gaussian beam in a saturable self-focusing medium
}

\author{
J. M. Soto-Crespo and E. M. Wright* \\ Optical Sciences Center, University of Arizona, Tucson, Arizona 85721 \\ N. N. Akhmediev \\ Institute of Physical Problems, Moscow 103460, U.S.S.R.
}

(Received 30 August 1991)

\begin{abstract}
In this paper we investigate the self-focusing of a Gaussian beam in a saturable nonlinear medium. We show that the dynamics of the incident Gaussian beam become progressively more complicated as the ratio of the Gaussian spot size to that of the ground-state solution is increased for a fixed energy flux. As this ratio is increased (or decreased) from unity, recurrence of the field is observed followed by a mixture of recurrence and azimuthal-symmetry breaking, and finally there is strong azimuthal-symmetry breaking resulting in beam breakup into solitary waves. In this last stage there is no evidence that the field will recur at larger propagation distances.
\end{abstract}

PACS number(s): 42.65.Jx, 52.35.Mw, 03.40.Kf, 03.65.Ge

\section{INTRODUCTION}

In 1968 Marburger and Dawes [1] published numerical simulations of the self-focusing of a cylindrical Gaussian beam in a nonlinear medium with a saturable, intensitydependent, refractive index. The significance of these simulations was that by including saturation they allowed the calculation to be carried through the first focus, in contrast to the nonsaturable case which predicted singular collapse [2-4], at least in the paraxial approximation [5]. These simulations showed that an incident Gaussian beam could go through multiple foci, sometimes periodically, with propagation distance $[1,4]$. This behavior was in agreement with the theoretical predictions of Wagner, Haus, and Marburger [6], obtained using a constantshape approximation. In general, however, the transverse-intensity profile of the propagating beam could develop a series of concentric-ring structures, in which case the constant-shape approximation was violated [1].

Later, Konno and Suzuki [7] reported numerical simulations of Gaussian beam self-focusing in a saturable nonlinear medium in which cylindrical symmetry of the propagating beam was not imposed from the outset as in previous studies $[1,3,4]$. Instead they solved the nonlinear propagation problem using Cartesian rather than cylindrical coordinates. They found that as the initial Gaussian beam propagates it can oscillate between a central focal spot and a ring structure, in agreement with Marburger and Dawes [1], but that upon further propagation the azimuthal symmetry could be broken (see Fig. 5 of Ref. [7]). This led to the situation in which the ring structure was replaced by four equally spaced focal spots. This is a clear example of a transverse instability. Transverse instabilities arise for nonlinear waves whose description requires fewer dimensions than that of the full geometry in which the problem is posed [2]. For the case at hand, the field can initially be described using the coordinates $(r, z)$, which is two dimensional. The trans- verse instability corresponds to growth of perturbations which depend on the azimuthal angle $\theta$ in threedimensional cylindrical coordinates, resulting in periodic beam breakup. Such periodic beam breakup had previously been investigated theoretically by Bespalov and Talanov [8], and Suydam [9], and both theoretically and experimentally by Campillo, Shapiro, and Suydam $[10,11]$ for the case of self-focusing in a Kerr nonlinear medium. For such a medium an input beam will typically breakup into several focal spots, except under wellcontrolled conditions when a single focal spot can arise [10].

In this paper we investigate the self-focusing of a Gaussian beam in a saturable nonlinear medium. Our incentive for studying this problem came from the fact that for a saturable nonlinear medium two types of interesting phenomena can arise. The first is recurrence in which an initial Gaussian beam can return very close to its initial profile many times during propagation (see Fig. 4 of Ref. [7]) $[1,4,7]$. (Recurrence has previously been studied in a number of other nonlinear wave equations with periodic boundary conditions imposed [12-18]). This cannot arise for a Kerr nonlinear medium. Second, periodic beam breakup resulting from azimuthal-symmetry breaking can occur, leading to the formation of focal spots, which can also arise for a Kerr nonlinear medium [10,11]. The key point is that for a saturable nonlinear medium recurrence can occur without the appearance of azimuthal-symmetry breaking. Konno and Suzuki appreciated this point as is clear from their paper, however, they did not give a physically transparent explanation of when to expect azimuthal-symmetry breaking [7]. Our goal in this paper is to provide a simple physical picture of the transition from recurrent behavior with cylindrical symmetry to azimuthal-symmetry breaking. Basically, the transition to azimuthal-symmetry breaking is associated with the appearance of a spatial ring in the field profile. An approximate stability analysis is presented 
which verifies that the symmetry breaking is indeed a transverse instability. In this way an intuitive criterion is established for when the results of simulations performed using cylindrical coordinates may be suspect due to azimuthal-symmetry breaking.

The remainder of this paper is organized as follows. Section II describes the nonlinear wave equation and notation we have employed and discusses the ground-state solution for a saturable nonlinear medium. In Sec. III we investigate the evolution of an initial Gaussian beam in the medium. In particular, we show how the field evolution varies as the input beam deviates further from the ground-state solution obtained in Sec. II, giving rise to both recurrence and azimuthal-symmetry breaking. Finally Sec. IV contains our summary and conclusions.

\section{BASIC THEORY AND GROUND-STATE SOLUTION}

In this section we describe the nonlinear wave equation used in this paper, and discuss the ground-state solution of the problem.

\section{A. Nonlinear wave equation}

We consider the propagation of a monochromatic, linearly polarized electric field of frequency $\omega$ in a saturable self-focusing medium characterized by the intensitydependent dielectric constant [19]

$$
\epsilon\left(|E|^{2}\right)=\epsilon_{b}+\Delta \epsilon_{\text {sat }} \frac{|E|^{2}}{\left(1+|E|^{2}\right)},
$$

where $\epsilon_{b}$ is the background dielectric constant, $\Delta \epsilon_{\mathrm{sat}}>0$ is the saturated change in dielectric constant, and $|E|^{2}$ is scaled such that it is the intensity in units of the saturation intensity. This particular form for the nonlinearity was chosen on the basis of its application to simple physical systems, namely, two-level systems, and in particular nonresonant excitation of atomic vapors [20-22]. In addition, this saturable nonlinearity does not give rise to catastrophic collapse in a finite distance [2]. Then, in the usual quasioptic approximations, and adopting the scaling and notation of Ref. [19], we obtain the following propagation equation for the slowly varying electric-field envelope $A$ :

$$
i \frac{\partial A}{\partial z}+\nabla_{T}^{2} A-q^{2} A+\frac{|A|^{2} A}{1+|A|^{2}}=0 .
$$

Here $q$ plays the role of a scaled effective index for the nonlinear wave, and the transverse Laplacian describing beam diffraction is

$$
\nabla_{T}^{2}=\frac{\partial^{2}}{\partial x^{2}}+\frac{\partial^{2}}{\partial y^{2}}=\nabla_{r r}^{2}+\frac{1}{r^{2}} \frac{\partial^{2}}{\partial \theta^{2}},
$$

in Cartesian and cylindrical coordinates, respectively, with

$$
\nabla_{r r}^{2}=\frac{\partial^{2}}{\partial r^{2}}+\frac{1}{r} \frac{\partial}{\partial r}
$$

Equation (2) is the starting point for our discussion of propagation in a saturable self-focusing medium.

\section{B. Ground-state solution}

The stationary solutions are defined by setting $\mathrm{\partial} A / \partial z=0$. If we concentrate on those stationary solutions with cylindrical symmetry $A=A(r)$, and assume $A$ is real without loss of generality, then Eq. (2) becomes
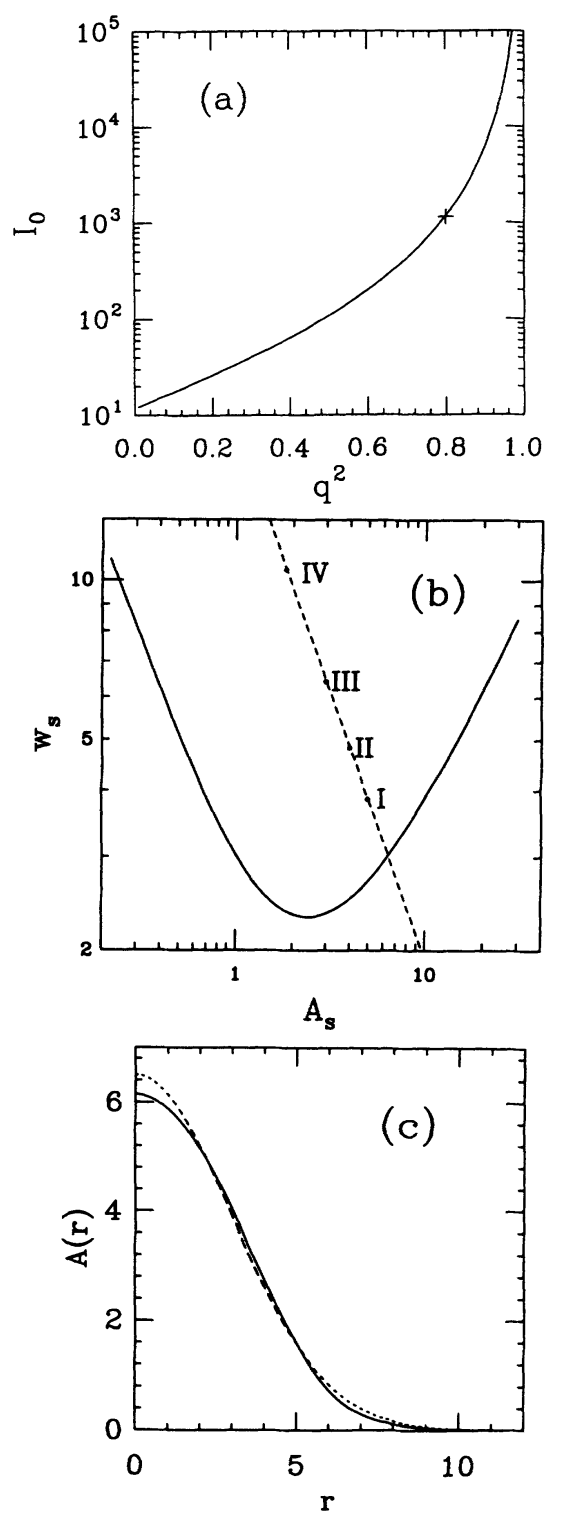

FIG. 1. (a) Calculated energy flux $I_{0}$ as a function of effective index $q^{2}$. The marked data point corresponds to the energy flux used in the following simulations, $I_{0}=1170.5$. (b) The solid curve shows the root mean square width $w_{s}$ of the stationary ground-state solution plotted against $A_{s}=A_{0}(0)$, and the dashed curve shows how the Gaussian beam waist $w_{G}$ (vertical axis) and on-axis field amplitude $A_{G}$ (horizontal axis) vary for a fixed flux of $I_{0}=1170.5$. The data points marked I-IV correspond to those used in the simulations: (I) $A_{G}=5, w_{G}=3.86$, (II) $A_{G}=4, w_{G}=4.82$, (III) $A_{G}=3, w_{G}=6.4$; and (IV) $A_{G}=1.85, w_{G}=10.4$. (c) Comparison between the exact ground-state solution (solid curve) for the data point marked in (a) $\left(I_{0}=1170.5, q^{2}=0.8\right)$, and the Gaussian approximation (8) with $A_{p}=6.5, w_{s}=2.97$ (dashed curve). 


$$
\nabla_{r r}^{2} A-q^{2} A+\frac{A^{3}}{1+A^{2}}=0
$$

with the boundary conditions

$$
\left.\frac{\partial A}{\partial r}\right|_{r=0}=\left.\frac{\partial A}{\partial r}\right|_{r \rightarrow \infty}=0, \quad A(r \rightarrow \infty)=0 .
$$

It is straightforward to show that $0 \leq q^{2} \leq 1$. Then for each allowed value of $q^{2}$, Eq. (5) has bound state solutions which we designate as $A_{n}, n=0,1,2, \ldots$, the $n$th solution having $n$ field nodes. For this paper we consider only the ground-state solution with $n=0$. We have obtained numerical solutions of Eqs. (5) using a shooting method, and for a given value of $q^{2}$ the corresponding ground-state field $A_{0}(r)$ was obtained. From these numerical solutions we then calculated the self-trapped energy flux

$$
I_{0}=\int_{0}^{\infty} 2 \pi r d r A_{0}^{2}(r)
$$

Figure 1(a) shows the calculated energy flux $I_{0}$ as a function of $q^{2}$. Vakhitov and Kolokolov [23] have shown that a sufficient condition for the stability of the ground state is $\partial I_{0} / \partial q^{2}>0$. With reference to Fig. 1(a) we see that the ground state is therefore stable.

Figure 1(b) shows an alternative classification of the ground-state solutions. For each value of $q$ in Fig. 1(a) we have calculated the root mean square width $w_{s}$ of the stationary ground state solution along with $A_{s}=A_{0}(0)$. The solid curve in Fig. 1(b) shows $w_{s}$ as a function of $A_{s}$, which was plotted parametrically using $q$ as the variable. The utility of this representation lies in the fact that the ground-state solution for a saturable medium can often be reasonably approximated as a Gaussian beam of the form $[6,22,24,25]$

$$
A_{0}(r) \simeq A_{p} \exp \left(-r^{2} / 2 s_{s}^{2}\right) \text {. }
$$

Figure 1(c) shows a comparison between the exact ground-state solution (solid curve) for the data point marked in Fig. 1(a) $\left(I_{0}=1170.5, q^{2}=0.8\right)$, and the Gaussian approximation (8) $\left(A_{p}=6.5, w_{s}=2.97\right.$, dashed curve). The Gaussian approximation is seen to give a good overall fit at the expense of slightly overestimating the on-axis field. This small shift in the on-axis field used in the Gaussian approximation is introduced so that the energy flux is the same as the exact ground state.

\section{NUMERICAL SIMULATIONS AND STABILITY ANALYSIS}

\section{A. Fixed numerical parameters}

The aim of the present section is to show the variety of behaviors that can arise when a Gaussian beam of the form

$$
A(r, 0)=A_{G} \exp \left(-r^{2} / 2 w_{G}^{2}\right)
$$

is propagated in a saturable nonlinear medium. For the simulations to be presented here we have fixed the in- cident energy flux to be $I_{G}=\pi\left|A_{G}\right|^{2} w_{G}^{2}=1170.5$, which is the same as the data point indicated in Fig. 1(a). The qualitative features of the conclusions drawn in the section are unaffected for other values of the energy flux as long as $I_{G}$ is a few times $I_{0}\left(q^{2}=0\right)$ [see Fig. 1(a)], $I_{0}\left(q^{2}=0\right)$ being the critical power for self-focusing in the absence of saturation.

The dashed curve in Fig. 1(b) shows how the Gaussian beam waist $w_{G}$ (vertical axis) varies with the on-axis field amplitude $A_{G}$ (horizontal axis) for a fixed energy flux of $I_{G}=1170.5$, and the particular choice $A_{G}=A_{p}$ and $w_{G}=w_{s}$ corresponds to the Gaussian approximation to the ground-state solution discussed in Sec. II B. For this energy flux the corresponding Gaussian approximation to the ground-state solution has $A_{p}=6.5$, and $w_{s}=2.97$. In the following discussion we shall present numerical simulations corresponding to the values of $A_{G}$ and $w_{G}$ labeled I-IV in Fig. 1(b). All of these data points have spot size $w_{G}$ larger than the ground state $w_{s}$. We have also performed numerical simulations with spot sizes smaller than the ground state, but the qualitative picture developed here remains the same.

\section{B. Numerical simulations in cylindrical coordinates}

First we shall discuss numerical simulations performed using two-dimensional cylindrical coordinates $(r, z)$, in which case the azimuthal symmetry of the problem is automatically preserved. For this case we solved the nonlinear wave equation with $\nabla_{T}^{2} \equiv \nabla_{r r}^{2}$ using a CrankNicholson scheme [26], along with the initial field given in Eq. (9). As a test of the scheme we propagated the Gaussian approximation to the ground-state solution for an energy flux $I_{G}=I_{0}=1170.5$. This initial field profile propagated stably over large distances with minimal change in profile, which is in accord with the fact that the exact ground-state solution is stable with respect to form (i.e., small transverse perturbations) [2]. The numerically determined ground-state solution propagated stably and with no detectable change in profile.

Figure 2 shows the results of simulations using a Gaussian input beam with $A_{G}=5$ and $w_{G}=3.86$ [point $I$ in Fig. 1(b)]. A phase-plane plot displaying $\operatorname{Im}[A(r=0, z)]$ versus $\operatorname{Re}[A(r=0, z)]$ is shown in Fig. 2(a), the curve being parametrized by the propagation coordinate $z$. Clearly the motion is highly periodic, and this is also displayed in Fig. 2(b) which shows the evolution of $|A(0, z)|^{2}$ as a function of $z$. This behavior is in complete agreement with the "small-signal" analysis of Wagner, Haus, and Marburger [6]. That is, since the ground-state solution represents a stable equilibrium point, a center, for small displacements of the input Gaussian beam from the ground state we expect that the Gaussian beam parameters will oscillate around the equilibrium values. In Fig. 2(a) this is seen as the small-radius oscillation, the net rotation around the origin being caused by linear and nonlinear phase modulation. In this case the propagating field periodically returns (very close) to the initial Gaussian beam thereby clearly displaying recurrence. 
The results of using Gaussian beamwidths which are still larger than the equilibrium value are shown in Figs. 3 and 4. Figures 3(a) and 4(a) show the phase-plane plots for $A_{G}=4$ and $w_{G}=4.82$ [point II in Fig. 1(b)], and $A_{G}=3$ and $w_{G}=6.4$ [point III in Fig. 1(b) ], respectively. In both cases the motion is still recurrent, though the degree of periodicity is clearly declining. (Note that, according to the broad meaning of recurrence due to Birkhoff [16], recurrence does not imply periodic motion or even quasiperiodic motion.) Figure 3(b) shows how the field profile $|A(r, z)|$ evolves with propagation distance for the smaller of the two beamwidths $w_{G}=4.82$. Note that the propagating field is starting to develop a shoulder in between those distances at which the field peaks up, and that there is clear evidence that radiation components are being generated. When the initial Gaussian beamwidth is further increased to $w_{G}=6.4$ this shoulder develops into a spatial ring which contains most of the beam energy flux. Figure 4(b) clearly shows this spatial ring (dashed curve) for a propagation distance of $z=24.2$, along with the initial Gaussian beam for reference (solid curve). In Fig. 4(a) the spatial ring is evidenced by the fact that the on-azis field twice passes close to zero, these corresponding to the propagation distances at which the spatial ring appears.
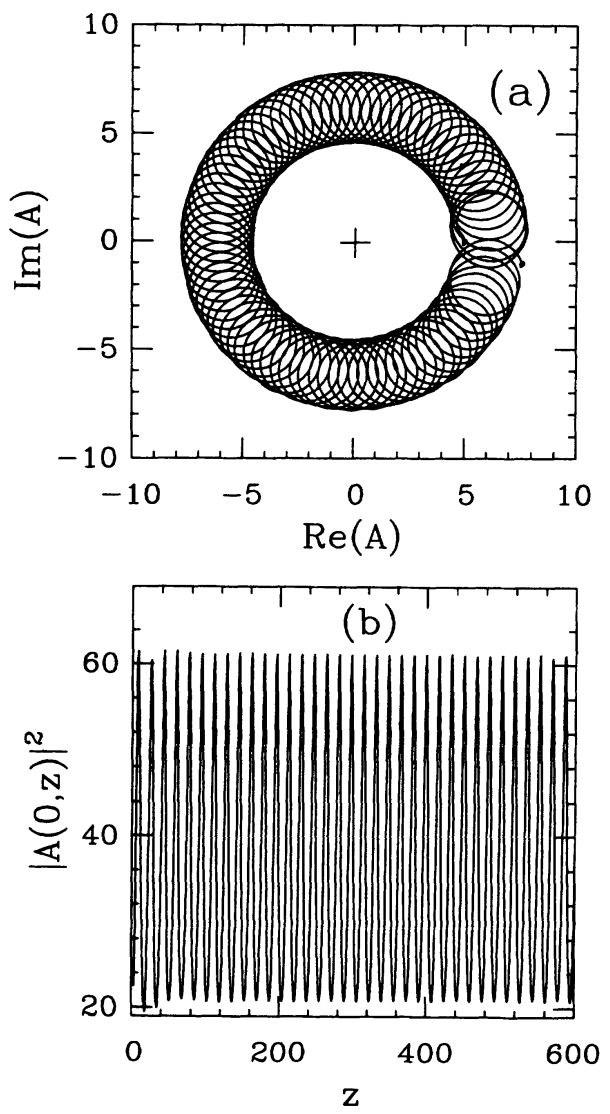

FIG. 2. (a) Phase-plane plot displaying $\operatorname{Im}[A(r=0, z)]$ vs $\operatorname{Re}[A(r=0, z)]$, the curve being parametrized by the propagation coordinate $z$, and (b) the evolution of $|A(0, z)|^{2}$ as a function of $z$, obtained using a Gaussian input beam with $A_{G}=5$ and $w_{G}=3.86$ [r oint $I$ in Fig. 1(b)].

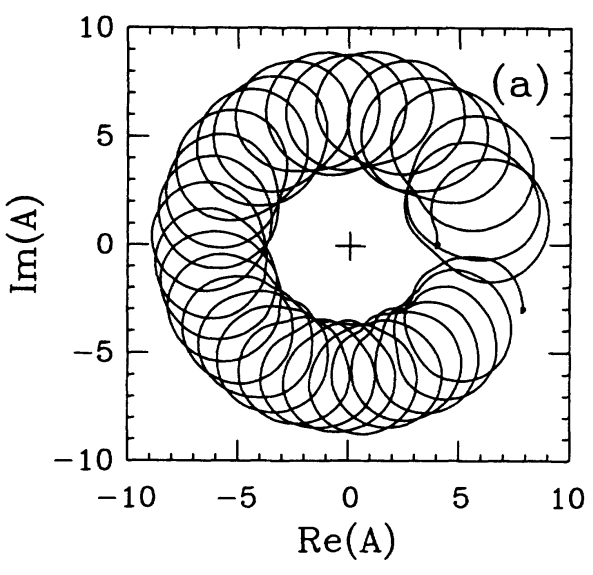

(b)

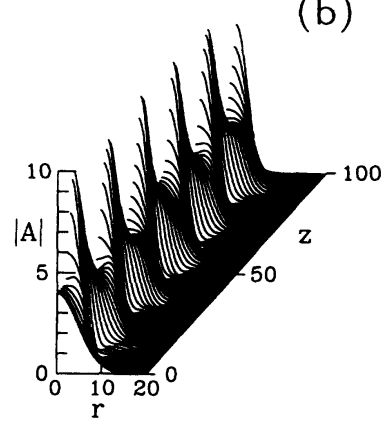

FIG. 3. (a) Phase-plane plot displaying $\operatorname{Im}[A(r=0, z)]$ vs $\operatorname{Re}[A(r=0, z)]$, the curve being parametrized by the progation coordinate $z$, and (b) the field profile $|A|$ as a function of radius $r$ and propagation distance $z$, obtained using a Gaussian input beam with $A_{G}=4$ and $w_{G}=4.82$ [point II in Fig. 1(b)].

For even larger initial Gaussian beamwidths the phase-plane plots become progressively less periodic and increasingly messy, and the propagating field profiles show the development of several rings in general $[1,7]$. For this study we are particularly interested in the case in which one ring appears as discussed above.

\section{Approximate stability analysis}

We now turn to the question of the stability of the cylindrically symmetric simulations. To proceed we first briefly describe our previous approach to the stability of the higher bound states of a saturable nonlinear medium $A_{n}(r), n=1,2,3, \ldots$ [19]. As mentioned in Sec. II B, the $n$th bound state has $n$ field nodes and its intensity profile consists of a central focal spot surrounded by $\boldsymbol{n}$ concentric spatial rings. For $n>0$ the higher bound states are stable in two-dimensional cylindrical coordinates $(r, z)$, but are unstable in three-dimensional cylindrical coordinates $(r, \theta, z)$, or Cartesian coordinates $(x, y, z)$. The propagating fields then develop azimuthal structure resulting in periodic beam breakup. This signals that a transverse instability is responsible for the instability of the higher bound states Since the bound states are stationary solutions of the nonlinear wave equation (2) a linear stability analysis could be performed around the invariant bound state profile. This was done numerically to 

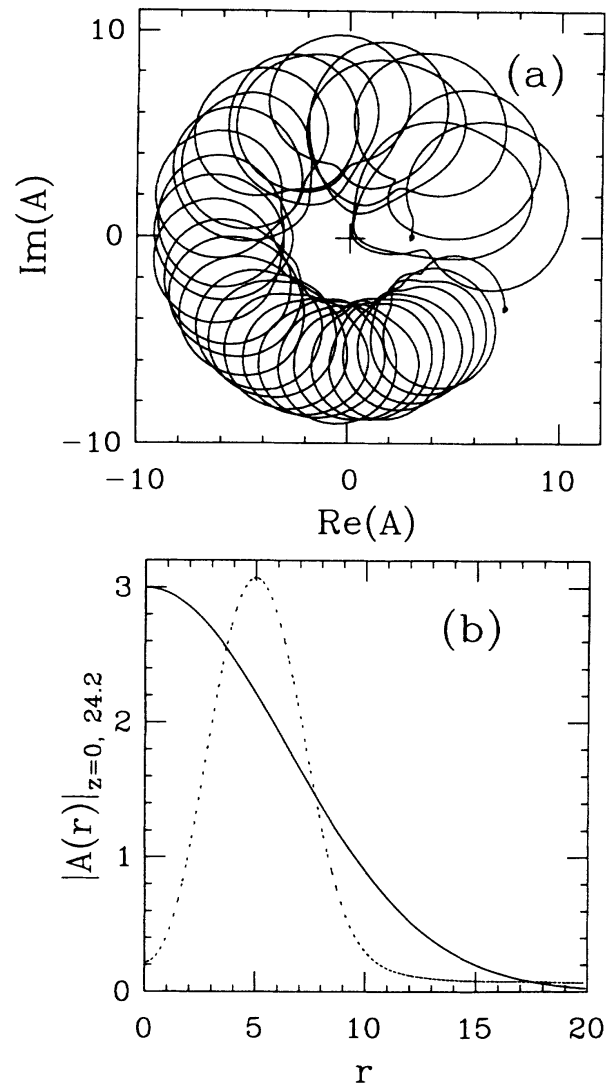

FIG. 4. (a) Phase-plane plot displaying $\operatorname{Im}[A(r=0, z)]$ vs $\operatorname{Re}[A(r=0, z)]$, the curve being parametrized by the propagation coordinate $z$, and (b) the field profile $|A(r, z)|$ at $z=0$ (solid curve) and $z=24.2$ (dashed curve), obtained using a Gaussian input beam with $A_{G}=3$ and $w_{G}=6.4$ [point III in Fig. 1(b)].

obtain the growth rates for the unstable perturbation eigenmodes. The results were then compared with those obtained from an approximate analytical stability theory, with excellent qualitative agreement. In this approximate theory the key idea was the stability properties could be assessed by considering each spatial ring separately. Then, for example, we were able to predict that for one example of an $n=2$ higher bound state the inner ring would break up into three focal spots, and the outer ring into seven focal spots (see Fig. 10 of Ref. [19]).

The point of the above discussion is to highlight the fact that the development of transverse instabilities is linked with the presence of spatial rings. Then, for example, for the simulations in Figs. 2 and 3 spatial rings are absent and we may therefore expect that these simulations would remain intact even if they were performed using Cartesian coordinates (which is indeed the case). In contrast, for the simulation in Fig. 4 the field does become a spatial ring at various stages of the propagation [Fig. 4(b)]. However, since the cylindrically symmetric field profile is not invariant along the propagation direction, in comparison to the higher bound states, a numerical stability analysis based on unstable perturbation eigenmodes is ruled out. Our approach will then be to apply the approximate analytical stability theory to the spatial ring in Fig. 4(b), the philosophy being that the transverse perturbation only grows when the field profile is in the vicinity of the spatial ring structure. (This highlights the importance of recurrence in the cylindrically symmetric problem to the development of transverse instabilities.) This approximate treatment for the instability growth rate neglects the transverse and longitudinal structures of the propagating field and must therefore be judged by the quality of the results it produces in comparison to the numerical solutions, which are given in the next section.

For this analysis we shall concentrate on the example shown in Fig. 4(b), since this is just within the regime in which transverse instability leading to azimuthalsymmetry breaking starts. That is, for initial Gaussian beam spot sizes $w_{G}<5$ symmetry breaking is not found to occur, or a spatial ring to form, whereas for $w_{G}>6$ several spatial rings may develop and symmetry breaking can occur in three-dimensional simulations (Sec. III D). We remark that this transition is not very sharp, it is in the range 5-6, and that where it occurs depends also on the initial beam flux (we have fixed $I_{0}=1170.5$ ). However, the qualitative features remain the same for other values of the beam flux.

Following the treatment in Ref. [19] we define the mean radius $\bar{r}$ and amplitude $\bar{A}$ of the spatial ring $A_{\mathrm{SR}}(r)$ in Fig. 4(b) as

$$
\bar{r}=\int 2 \pi r^{2}\left|A_{\mathrm{SR}}(r)\right|^{2} d r / \int 2 \pi r\left|A_{\mathrm{SR}}(r)\right|^{2} d r,
$$

and

$$
\bar{A}=\int 2 \pi r\left|A_{\mathrm{SR}}(r)\right|^{3} d r / \int 2 \pi r\left|A_{\mathrm{SR}}(r)\right|^{2} d r,
$$

where the integrations extend over the numerical grid. For this case $\bar{r}=5.675$ and $\bar{A}=2.49$. We now assume that the stability properties of the spatial ring can be obtained from a simple one-dimensional model in which the ring of radius $\bar{r}$ is unfolded. The equation for the field amplitude $A(s, z)$ along the ring is taken of the form

$$
i \frac{\partial A}{\partial z}+\frac{\partial^{2} A}{\partial s^{2}}-q^{2} A+\frac{|A|^{2} A}{1+|A|^{2}}=0,
$$

where $s$ is the path length along the ring, and

$$
q^{2}=\frac{\bar{A}^{2}}{1+\bar{A}^{2}} .
$$

We have chosen this value of the effective-index $q^{2}$ so that $A=\bar{A}$ is a stationary solution of Eq. (12). The idea is to obtain the growth rate of perturbations while the field profile is in the vicinity of the spatial ring. Notice that in this picture the spatial ring is simply represented by the setting $A=\bar{A}$ at all points around the ring. We investigate the stability of this solution by setting

$$
A(s, z)=\bar{A}+\mu \cos (m \theta) \exp \left(\delta_{m} z\right),
$$

where we have used a $s=\bar{r} \theta / 2 \pi$ in Eq. (12), $m$ is the azimuthal index, $\mu$ is a small constant, and $\delta_{m}$ is the growth rate. Note that the form chosen for the perturbation in 
Eq. (14) ensures that the field is periodic in $\theta$, but allows for modulations to develop around the ring. This means that we are dealing with a transverse instability which breaks the azimuthal symmetry of the problem [2,9-11,19]. By substituting Eq. (14) into (12), linearizing with respect to $\mu$, and solving for $\delta_{m}$ we obtain

$$
\delta_{m}=\left(\frac{m}{\bar{r}}\right]\left[\frac{2 \bar{A}^{2}}{\left(1+\bar{A}^{2}\right)^{2}}-\left(\frac{m}{\bar{r}}\right)^{2}\right]^{1 / 2}
$$

Figure 5 shows the predicted growth rate $\delta_{m}$ as a function of the azimuthal index $m$ for the spatial ring in Fig. 4(b), $\bar{r}=5.675$ and $\bar{A}=2.49$ (only the integer values of $m$ are physically relevant). Here we clearly see that the largest growth rate occurs for $m=2$. This means that in a three-dimensional simulation we expect that the initial cylindrical symmetry of this example will be broken to a twofold symmetry upon propagation.

\section{Numerical simulations in Cartesian coordinates}

In the section we present some numerical simulations of the nonlinear wave equation (2) in Cartesian coordinates. In particular, we have solved Eq. (2) using the split-step method [27] on a Cartesian grid with the initial condition

$$
A(x, y, 0)=A_{G} \exp \left[-\left(x^{2}+y^{2}\right) / 2 w_{G}^{2}\right][1+\Gamma(x, y)],
$$

where $\Gamma$ is a $\delta$-correlated white-noise source obeying

$$
\langle\Gamma(x, y)\rangle=0, \quad\left\langle|\Gamma(x, y)|^{2}\right\rangle=\frac{1}{100} .
$$

The noise term is intended to mimic random fluctuations in the input beam which will serve to seed any latent instability in the system. All of the numerical simulations reported here were verified for several realizations of the noise term to ensure that our conclusions were not the product of a wild fluctuation.

Simulations using Cartesian coordinates have verified that for $w_{G}<5$ there is no transverse instability leading to symmetry breaking even for very large propagation distances, typically $z \simeq 500$. That is, the results shown in

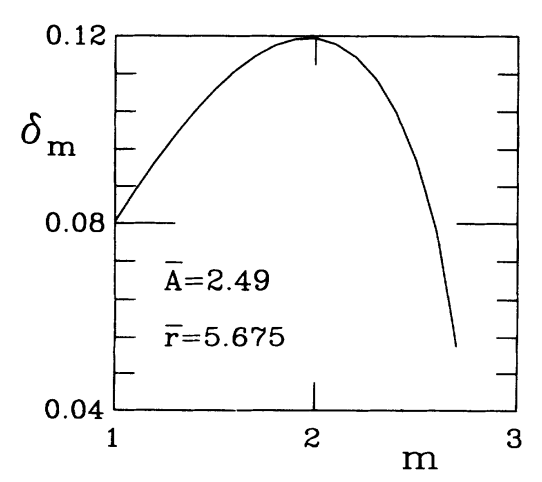

FIG. 5. Predicted growth rate $\delta_{m}$ as a function of the azimuthal index $m$ for the spatial ring in Fig. $4(b), \bar{r}=5.675$ and $\bar{A}=2.49$. Only the integer values of $m$ are physically relevant.
Figs. $2\left(w_{G}=3.8\right)$ and $3\left(w_{G}=4.8\right)$ are independent whether a two- or three-dimensional coordinate system is used. This does not, of course, constitute a proof, since the growth rate could just be very slow. However, as shown in Fig. 6, for $w_{G}=6.4$, corresponding to the case in Fig. 4 [point III in Fig. 1(b)], the propagating field now displays azimuthal-symmetry breaking for a propagation distance of only $z=90$. This figure shows the field intensity profiles as a function of the transverse coordinates $(x, y)$ at selected propagation distances. As the field propagates it initially goes between a central focal spot and a spatial ring as illustrated in Fig. 4. However, upon further propagation the continuous rotational symmetry of the spatial ring is broken yielding the twofold symmetric structure shown in Fig. 6(a) $(z=90)$. [For different realizations of the noisy input beam (16) the same twofold symmetry is observed but the orientation of the structure varies.] This is in exact agreement with the analytic prediction of Sec. III C. As the field propagates further it returns again to a central focal spot. Figure 6(b) illustrates how the twofold symmetric structure looks the next time it appears at $z=107.5$. Here we see that the azimuthal modulation continues to grow with propagation distance as expected, however, a $90^{\circ}$ rotation of the orientation of the twofold symmetric structure is observed. One possible explanation for this phenomenon is that it is a nonlinear Berry's phase arising from the fact that the system is going through near cyclic motions [28]. We are currently investigating this phenomenon in more detail.
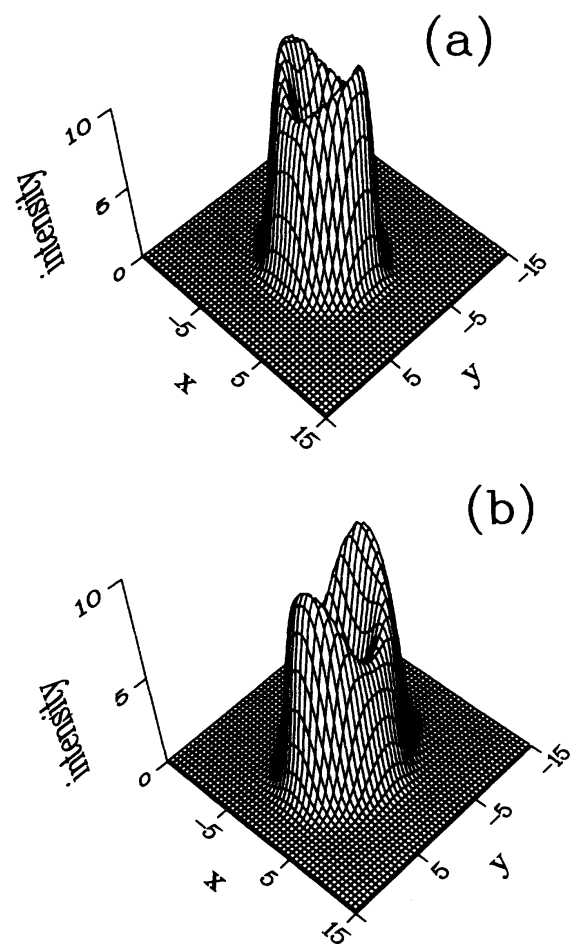

FIG. 6. Field intensity profiles $|\boldsymbol{A}|^{2}$ as a function of the transverse coordinates $(x, y)$ for (a) $z=90$, and (b) $z=107.5$. The simulation was performed with $A_{G}=3$ and $w_{G}=6.4$ [point III in Fig. 1(b)]. 

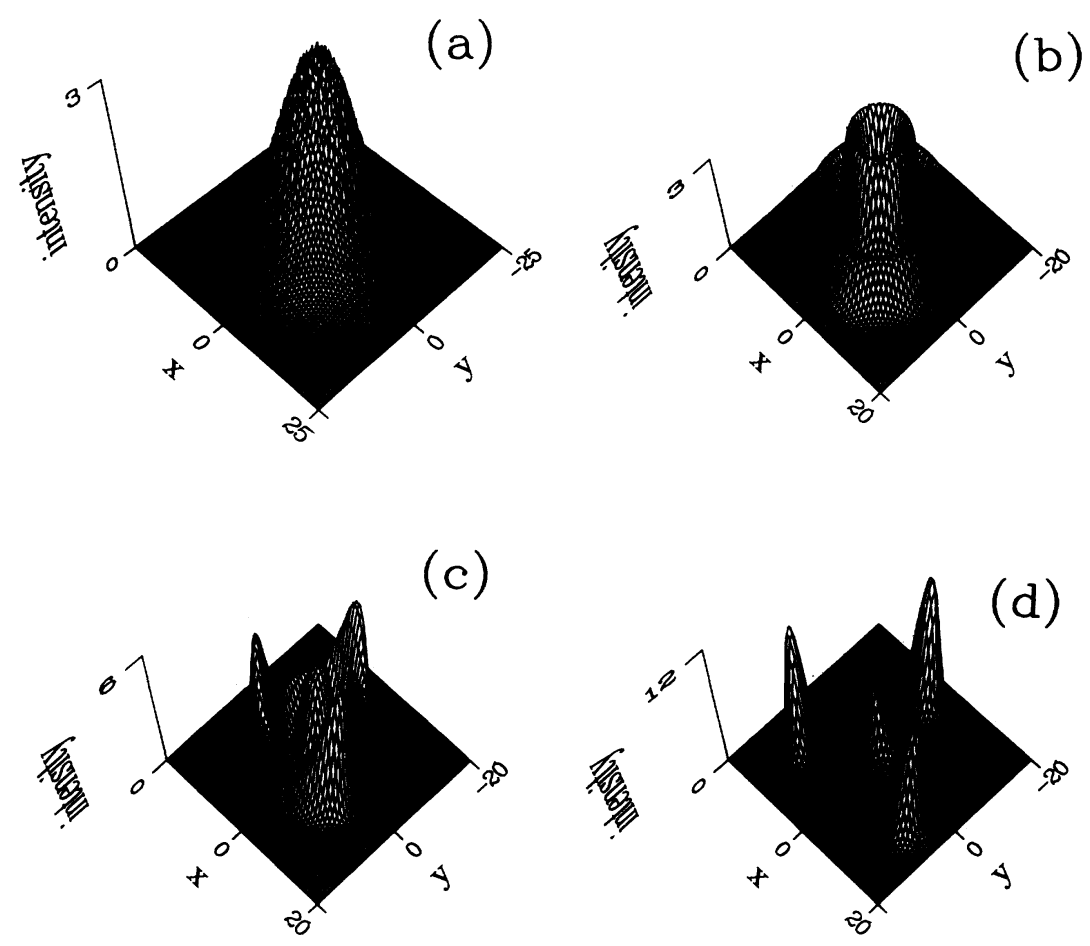

FIG. 7. Field intensity profiles $|A|^{2}$ as a function of the transverse coordinates $(x, y)$ for (a) $z=0$, (b) $z=30$, (c) $z=60$, and (d) $z=90$. The simulation was performed with $A_{G}=1.85$ and $w_{G}=10.4$ [point IV in Fig. 1(b)].

As the initial Gaussian beam spot size is further increased several spatial rings may develop, and the transverse instability develops faster. This is illustrated in Fig. 7 which shows a series of intensity profiles as a function of propagation distance for $w_{G}=10.4$ [point VI in Fig. 1(b)]. Here we see that the initial Gaussian beam [Fig. 7(a)] now distorts into a pair of spatial rings [Fig. 7(b)]. Upon further propagation the profile evolves into a central focal spot and a threefold symmetric structure [Fig. $7(\mathrm{c})]$. For a propagation distance of $z=90^{\circ}[\mathrm{Fig} \mathrm{7(d)]} \mathrm{the}$ field is seen to be composed of four focal spots which retain their profiles but propagate apart with further propagation. This verifies that the four focal spots in this case are spatial solitary waves. It appears in this case that there is no longer any recurrence since the individual solitary waves appear to separate with no sign of slowing down.

Konno and Suzuki first showed that an initial Gaussian beam can decay into a set of solitary waves [7]. However, their numerics produced a spatial pattern with fourfold symmetry which would seem to implicate their numerical grid in seeding the spontaneous symmetry breaking. On the other hand, by introducing noise into the input field profile we have essentially swamped the grid noise so that the unstable mode of highest growth rate will dominate. In this way we are able to see the predominantly two- and threefold symmetries evident in Figs. 6 and 7. More recently Grantham et al. [29] have shown that by applying appropriate symmetry breaking perturbations to the input beam the field profiles transmitted at the cell exit of sodium can display bifurcations in the solitary-wave patterns. In their case the effects of input beam noise are swamped by the applied perturbations which makes for more stable experimental observations. Their bifurcations are therefore not a result of spontaneous symmetry breaking but rather induced symmetry breaking. In contrast, this work has been concerned only with patterns arising from spontaneous symmetry breaking.

\section{SUMMARY AND CONCLUSIONS}

In this paper we have investigated the self-focusing of a Gaussian beam in a saturable nonlinear medium. We have shown that the dynamics of the incident Gaussian beam become progressively more complicated as the ratio of the Gaussian spot size to that of the ground-state solution is increased for a fixed energy flux. As this ratio is increased (or decreased) from unity recurrence of the field $[1,7]$ is observed followed by a mixture of recurrence and azimuthal-symmetry breaking, and finally there is strong azimuthal-symmetry breaking resulting in beam breakup into solitary waves $[7,29]$. In this last stage there is no evidence that the field will recur at larger propagation distances.

This work provides a simple physical picture of the transition from recurrent behavior with cylindrical symmetry to azimuthal-symmetry breaking. We have argued that the transition to azimuthal-symmetry breaking is associated with the appearance of a spatial ring in the field profile, and this was verified using an approximate stability analysis of the transverse instability. A clear indicator 
that two-dimensional $(r, z)$ simulations of the nonlinear wave equation may be suspect and that full threedimensional $(r, \theta, z$ or $x, y, z)$ simulations are required is that spatial rings start to develop. These results may also be of use in related problems such as pattern formation and symmetry breaking in lasers [30-32].

\section{ACKNOWLEDGMENTS}

J. M. Soto-Crespo acknowledges a grant from the Ministerio de Educacion y Ciencia of Spain. The authors would like to thank Dr. David R. Heatley for several useful discussions regarding this work.
*Also with the Physics Department, University of Arizona, Tucson, AZ 85721.

[1] J. H. Marburger and E. Dawes, Phys. Rev. Lett. 21, 556 (1968); Phys. Rev. 179, 862 (1969).

[2] For a review of self-focusing instabilites see, e.g., J. J. Rasmussen and K. Rypdal, Phys. Scr. 33, 481 (1986), and references therein.

[3] P. Kelley, Phys. Rev. Lett. 15, 1005 (1965).

[4] V. E. Zakharov, V. V. Sobolev, and V. C. Synakh, Zh. Eksp. Teor. Fiz. 60, 136 (1971) [Sov. Phys._JETP 33, 77 (1971)].

[5] M. D. Feit and J. A. Fleck, J. Opt. Soc. Am. B 5, 633 (1988).

[6] W. G. Wagner, H. A. Haus, and J. H. Marburger, Phys. Rev. 175, 256 (1968).

[7] K. Konno and H. Suzuki, Phys. Scr. 20, 382 (1979).

[8] V. I. Bespalov and V. I. Talanov, Pis'ma Zh. Eksp. Teor. Fiz. 3, 471 (1966) [JETP Lett. 3, 307 (1966)].

[9] B. R. Suydam, IEEE J. Quantum Electron. QE-11, 225 (1975).

[10] A. J. Campillo, S. L. Shapiro, and B. R. Suydam, Appl. Phys. Lett. 23, 628 (1973).

[11] A. J. Campillo, S. L. Shapiro, and B. R. Suydam, Appl. Phys. Lett. 24, 178 (1974).

[12] E. Fermi, J. Pasta, and S. Ulam, in Collected Papers of Ernico Fermi, edited by E. Segre (University of Chicago Press, Chicago, 1965), Vol. 2, p. 978.

[13] N. J. Zabusky and M. D. Krushkal, Phys. Rev. Lett. 15, 240 (1965).

[14] H. C. Yuen and W. E. Ferguson, Jr., Phys. Fluids 21, 1275 (1978).

[15] H. C. Yuen and W. E. Ferguson, Jr., Phys. Fluids 21, 2116 (1978).

[16] A. Thyagaraja, Phys. Fluids 22, 2093 (1979).

[17] A. Thyagaraja, in Nonlinear Waves, edited by L. Debnath
(Cambridge University Press, London, 1983), Chap. 17.

[18] N. N. Akhmediev, D. R. Heatley, G. I. Stegeman, and E. M. Wright, Phys. Rev. Lett. 65, 1423 (1990).

[19] J. M. Soto-Crespo, D. R. Heatley, E. M. Wright, and N. N. Akhmediev, Phys. Rev. A 44, 636 (1991).

[20] D. Grischkowsky, Phys. Rev. Lett. 24, 866 (1970).

[21] J. E. Bjorkholm and A. Ashkin, Phys. Rev. Lett. 32, 129 (1974).

[22] M. Le Berre, E. Ressayre, A. Tallet, and F. P. Mattar, J. Opt. Soc. Am. B 2, 956 (1985).

[23] N. G. Vakhitov and A. A. Kolokolov, Izv. Vyssh. Uchebn. Zaved. Radiofiz. 16, 1020 (1973) [Sov. Radiophys. 9, 261 (1966)]; A. A. Kolokolov, ibid. 17, 1332 (1974) [ibid. 17, 1016 (1976)].

[24] D. Anderson, M. Bonnedal, and M. Lisak, Phys. Fluids 22, 1838 (1979).

[25] Y. Chen, Opt. Lett. 16, 4 (1991).

[26] W. H. Press, B. P. Flannery, S. A. Teukolsky, and W. T. Vetterling, Numerical Recipes (Cambridge University Press, Cambridge, 1986), p. 640.

[27] J. A. Fleck, J. R. Morris, and M. D. Feit, J. Appl. Phys. 10, 129 (1975); M. D. Feit and J. A. Fleck, J. Opt. Soc. Am. B 5, 633 (1988).

[28] J. C. Garrison and R. Y. Chiao, Phys. Rev. Lett. 60, 165 (1988).

[29] J. W. Grantham, H. M. Gibbs, G. Khitrova, J. F. Valley, and Xu Jiajin, Phys. Rev. Lett. 66, 1422 (1991).

[30] L. A. Lugiato, G. L. Oppo, J. R. Tredice, and L. M. Narducci, J. Opt. Soc. Am. B 7, 1019 (1990).

[31] L. A. Lugiato, F. Prati, L. M. Narducci, and G. L. Oppo, Opt. Commun. 69, 387 (1989).

[32] M. Brambilla, F. Battipede, L. A. Lugiato, V. Penna, F. Prati, C. Tamm, and C. O. Weiss, Phys. Rev. A 43, 5090 (1990). 

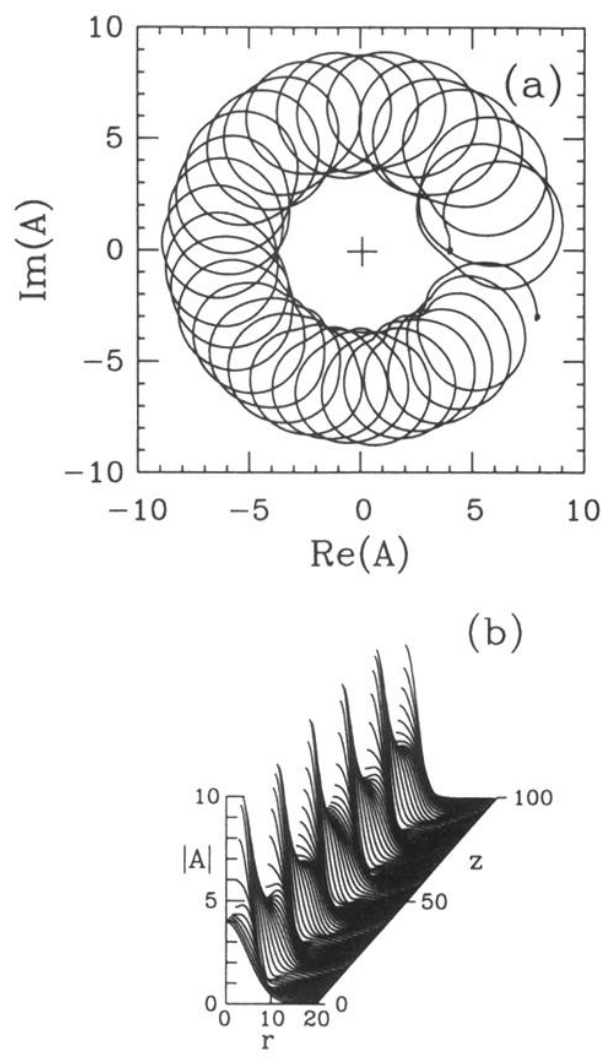

FIG. 3. (a) Phase-plane plot displaying $\operatorname{Im}[A(r=0, z)]$ vs $\operatorname{Re}[A(r=0, z)]$, the curve being parametrized by the progation coordinate $z$, and (b) the field profile $|A|$ as a function of radius $r$ and propagation distance $z$, obtained using a Gaussian input beam with $A_{G}=4$ and $w_{G}=4.82$ [point II in Fig. 1(b)]. 


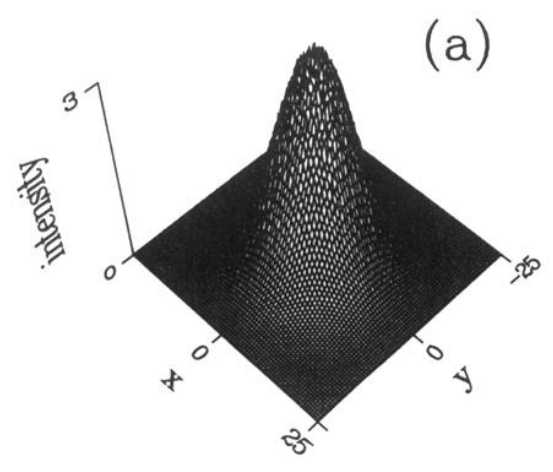

(b)
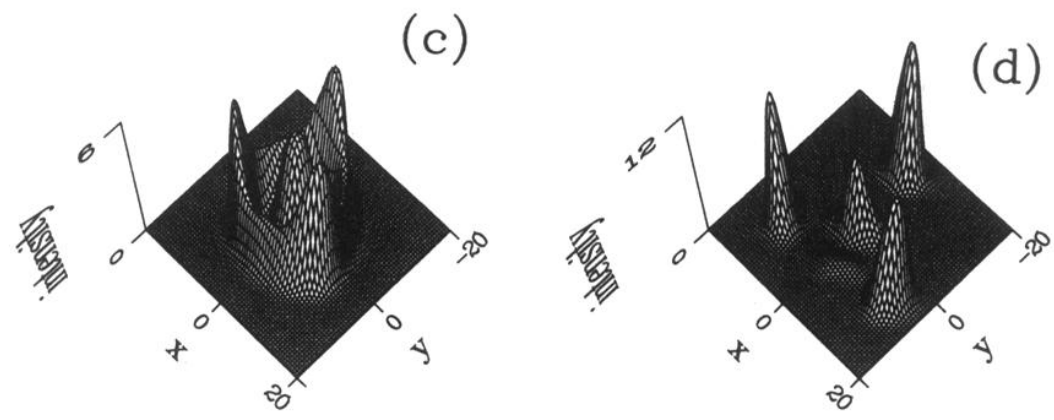

FIG. 7. Field intensity profiles $|A|^{2}$ as a function of the transverse coordinates $(x, y)$ for (a) $z=0$, (b) $z=30$, (c) $z=60$, and (d) $z=90$. The simulation was performed with $A_{G}=1.85$ and $w_{G}=10.4$ [point IV in Fig. 1(b)]. 